\title{
Long term lightcurve of the BL Lac object 1ES 0229+200 at TeV energies
}

\author{
Gabriele Cologna $^{* a} \dagger$, Mahmoud Mohamed ${ }^{a \dagger}$, Stefan J. Wagner ${ }^{a}$, Alicja Wierzcholska ${ }^{a \dagger}$, \\ Carlo Romoli $^{b}$ for the H.E.S.S. Collaboration and Omar Kurtanidze ${ }^{c, a}$ \\ ${ }^{a}$ Landessternwarte, Universität Heidelberg, Königstuhl, D 69117 Heidelberg, Germany \\ ${ }^{b}$ Dublin Institute for Advanced Studies, 31 Fitzwilliam Place, Dublin 2, Ireland \\ ${ }^{c}$ Abastumani Observatory, Mt. Kanobili, 0301 Abastumani, Georgia \\ E-mail: gcologna@lsw. uni-heidelberg.de
}

The high-frequency peaked BL Lac object 1ES 0229+200 ( $\mathrm{z}=0.14)$ was first detected in very high energy (VHE, E $>100 \mathrm{GeV}) \gamma$-rays by the H.E.S.S. (High Energy Stereoscopic System) collaboration in 2006 [1]. No flux variability was reported in the initial study and its spectral characteristics have been used to derive constraints on the extragalactic background light (EBL, [1]) and on the intergalactic magnetic field (IGMF, e.g. [2, 3, 4, 5]). 1ES 0229+200 has been observed with H.E.S.S. for $\sim 130$ hours from 2004 to 2013: the full dataset analysed with a more sensitive method will be presented here. The results indicate that the source is not constant and displays flux variability on yearly and monthly timescales. The existence of flux variability affects the derivation of the constraints on the IGMF. The H.E.S.S. observations cover several simultaneous multi-frequency campaigns and the VHE variations are compared with those reported in different bands.

The 34th International Cosmic Ray Conference,

30 July- 6 August, 2015

The Hague, The Netherlands

\footnotetext{
*Speaker.

${ }^{\dagger}$ Member of the International Max Planck Research School for Astronomy and Cosmic Physics at the University of Heidelberg (IMPRS-HD) and the Heidelberg Graduate School of Fundamental Physics (HGSFP).

${ }^{\ddagger}$ Mobility Plus Fellow
} 


\section{Introduction}

1ES $0229+200$ is a blazar, and a member of the high frequency peaked BL Lac objects (HBL) subclass. It is hosted in an elliptical galaxy located at a redshift $z=0.1396$. It was first discovered in X-rays by the Einstein IPC Slew Survey in 1992 [6]. Its synchrotron emission reaches $100 \mathrm{keV}$ and possibly up to $200 \mathrm{keV}^{1}$. Despite being relatively bright in optical and X-rays, it is faint in radio and $\gamma$-rays. It has been detected at VHE above $500 \mathrm{GeV}$ for the first time by H.E.S.S. in 2006 [1], and only in 2011 at high energies (HE, $100 \mathrm{MeV}<\mathrm{E}<100 \mathrm{GeV}$ ) between 1 and $300 \mathrm{GeV}$, after more than three years of Fermi observations [5]. Contrary to what one could expect from its blazar nature, 1ES 0229+200 shows little variability at all energies, even if it is known to have flux variations in the $\mathrm{X}$-ray band of a factor of $\sim 2$. (e.g. [7]). Hints of variability at $\mathrm{TeV}$ energies were reported on yearly and monthly timescales by the VERITAS collaboration in 2014 [8], while none was detected in the source in the initial H.E.S.S. study.

1ES $0229+200$ has been one of the key sources for deriving constraints on the extragalactic background light (EBL, [1]) and on the intergalactic magnetic field (IGMF, e.g. [2, 3, 4, 5]) thanks to the combination of its hard spectrum $\left(\Gamma_{P L} \sim 2.5,[1,8]\right)$ reaching $\sim 10 \mathrm{TeV}$ and its considerable distance (for a $\mathrm{TeV}$ source). The spectrum allows one to probe the near- and mid-infrared wavelengths of the EBL $(\sim 2-20 \mu \mathrm{m})$ at high optical depths $(\tau \sim 1-6$ for $\gamma$-ray energies between $\sim 1-10 \mathrm{TeV})$. This is unique, since only a few other sources have been observed at these energies. For the IGMF studies, a multi-TeV hard spectrum in combination with high optical depths implies that a considerable part of the emitted $\mathrm{TeV}$ photons will be absorbed via pair production through their interaction with the EBL and re-emitted at (typically) $\mathrm{GeV}$ energies, causing a surplus of $\mathrm{HE}$ emission. Lower limits on the strength of the IGMF can be calculated measuring the $\mathrm{GeV}$ emission and making assumptions on the intrinsic spectrum. Since reprocessing involves emission from an ensemble of charged particles that are spread over an extended volume by the action of the magnetic field, time delays are of the order of the light travel time of the excess distance that the re-emitted photons must cover with respect to the line of sight to the source. Time delays are also energy dependent, since a given magnetic field will affect more strongly the trajectories of low-energy particles. This holds true as long as the Compton cooling time is of the order of the gyroradius. If it is too fast, re-emission is almost immediate and time delay negligible. If it is too slow, this and not the magnetic field determines the time delay. The observed reprocessed emission corresponds therefore to the time-averaged original VHE emission. Constrains on the IGMF hence rely on the assumption of a steady VHE flux on time scales of these delays. Is the VHE flux variable, average values can be used as long as the variability is shorter than the reprocessing time delay.

\section{Observations and Analysis}

\subsection{H.E.S.S.}

H.E.S.S. observations have been carried out almost yearly between 2004 and 2013, for a total of 354 observation runs ${ }^{2} .311$ of them pass the standard quality cuts for a total livetime of $\sim 133 \mathrm{~h}$

\footnotetext{
${ }^{1}$ http://bat.ifc.inaf.it/100m_bat_catalog/100m_bat_catalog_v0.0.htm

${ }^{2}$ The term "run" refers to a single observation, with a typical exposure of 28 minutes.
} 
Table 1: Statistics of the yearly and total H.E.S.S. observations.

\begin{tabular}{c|ccccccc}
\hline \hline Period & $\begin{array}{c}\text { Dates } \\
(\mathrm{MJD})\end{array}$ & $\begin{array}{c}\text { Live Time } \\
(\mathrm{h})\end{array}$ & $\begin{array}{c}\text { ON } \\
\text { counts }\end{array}$ & $\begin{array}{c}\text { OFF } \\
\text { counts }\end{array}$ & $\begin{array}{c}\text { excess } \\
(\sigma)\end{array}$ & $\begin{array}{c}\text { Significance } \\
(\sigma)\end{array}$ & $\begin{array}{c}\text { Flux }(>580 \mathrm{GeV}) \\
\left(10^{-13} \mathrm{~cm}^{-2} \mathrm{~s}^{-1}\right)\end{array}$ \\
\hline \hline $2004-2013$ & $53259-56606$ & 132.5 & 1839 & 14138 & 699 & 18.1 & $6.2 \pm 0.5$ \\
\hline 2004 & $53259-53317$ & 3.8 & 47 & 349 & 18 & 2.9 & $3.5 \pm 1.9$ \\
2005 & $53613-53649$ & 16.4 & 169 & 1518 & 42 & 3.4 & $2.5 \pm 1.0$ \\
2006 & $53967-54088$ & 47.1 & 750 & 4577 & 368 & 15.8 & $9.0 \pm 0.8$ \\
2007 & $54322-54336$ & 10.5 & 137 & 1080 & 47 & 4.4 & $5.6 \pm 1.7$ \\
2008 & $54681-54789$ & 12.2 & 149 & 1420 & 31 & 2.6 & $4.0 \pm 1.6$ \\
2009 & $55063-55151$ & 19.0 & 215 & 1825 & 63 & 4.6 & $5.2 \pm 1.4$ \\
2011 & $55801-55909$ & 7.4 & 112 & 918 & 35 & 3.6 & $1.9 \pm 2.0$ \\
2013 & $56514-56606$ & 17.8 & 241 & 2189 & 95 & 6.9 & $7.4 \pm 1.3$ \\
\hline \hline
\end{tabular}

Note: the normalization factor $\beta$ between the ON and OFF area is 1/12 for all years with the exception of 2013 1/15.

( $122 \mathrm{~h}$ when corrected for acceptance). The mean zenith angle is $45.2^{\circ}$ and the mean offset from the pointing position $0.51^{\circ}$. The exposure was not homogeneous during these observation years, ranging from $3.8 \mathrm{~h}$ in 2004 to $47.1 \mathrm{~h}$ in 2006 . Refined quality selection criteria allowed for a larger dataset for the years 2004-2006 than the one published in [9] and [1]. Part of the 2009 and 2013 data belong to multiwavelength (MWL) campaigns. The first one was organized with XMMNewton and ATOM on August 21 and 23, 2009, the second one with NuSTAR, Swift-XRT, MAGIC and VERITAS on October 1, 5 and 11,2013. Data reduction has been performed using the Model analysis $^{3}$ [10] with Standard cuts. For the background determination for the spectral and temporal analyses, the Reflected Region Background method [11] was used. The source is detected with a significance of 18.1 standard deviations $(\sigma)$ for the whole dataset. The results for the total dataset and for every year of observation can be found in Tab.1, as well as in Tab.2 for the 2006 and 2009 observing periods.

Spectral analyses have been carried out on different data subsets using the forward-folding technique [12] and a simple power-law (PL) spectral shape. No spectral variability is detected on a yearly nor monthly timescale. The lightcurves are derived adjusting the normalization of a PL with index $\Gamma=2.9$ to the $\gamma$-ray excess in every time bin. Only energies above a common threshold of $580 \mathrm{GeV}$ are considered. The monthly and yearly lightcurves are shown in the top two panels of Fig. 1 and discussed in Sec. 3. All results have been cross checked with a different software and calibration chain.

\subsection{High Energies}

HE data have been collected by the Large Area Telescope (LAT) onboard the Fermi satellite starting August 4, 2008. Data up to April 27, 2015 have been analysed with the ScienceTool software package version v9r33p0. Only events belonging to the 'Source' class within $15^{\circ}$ from the position of $1 \mathrm{ES} 0229+200$ were selected. Moreover, cuts on zenith angle $\left(100^{\circ}\right)$,

\footnotetext{
${ }^{3}$ software version paris-0-8-24, DSTs Prod26
} 
Table 2: Statistics of the 2006 and 2009 observation periods.

\begin{tabular}{l|ccccccc}
\hline \hline \multirow{2}{*}{ Period } & $\begin{array}{c}\text { Dates } \\
(\mathrm{MJD})\end{array}$ & $\begin{array}{c}\text { Live Time } \\
(\mathrm{h})\end{array}$ & $\begin{array}{c}\text { ON } \\
\text { counts }\end{array}$ & $\begin{array}{c}\text { OFF } \\
\text { counts }\end{array}$ & $\begin{array}{c}\text { excess } \\
(\sigma)\end{array}$ & $\begin{array}{c}\text { Significance } \\
(\sigma)\end{array}$ & $\begin{array}{c}\text { Flux }(>580 \mathrm{GeV}) \\
\left(10^{-13} \mathrm{~cm}^{-2} \mathrm{~s}^{-1}\right)\end{array}$ \\
\hline \hline & & & \multicolumn{7}{c}{2006} \\
\cline { 2 - 8 } August & $53967-53977$ & 13.5 & 226 & 1581 & 94 & 7.1 & $8.1 \pm 1.6$ \\
September & $53994-54005$ & 16.0 & 329 & 1813 & 178 & 11.9 & $12.2 \pm 1.6$ \\
November & $54048-54063$ & 12.5 & 146 & 813 & 78 & 7.8 & $6.9 \pm 1.4$ \\
December & $54077-54088$ & 5.1 & 49 & 370 & 18 & 2.9 & $6.8 \pm 2.0$ \\
\hline \hline & & & \multicolumn{7}{c}{2009} & & \\
August & $55063-55074$ & 9.3 & 72 & 815 & 4 & 0.5 & $-0.5 \pm 1.5$ \\
October & $55115-55121$ & 5.1 & 73 & 561 & 26 & 3.4 & $7.0 \pm 2.9$ \\
November & $55145-55151$ & 4.6 & 70 & 449 & 33 & 4.5 & $16.6 \pm 3.8$ \\
\hline \hline
\end{tabular}

Note: the normalization factor $\beta$ between the ON and OFF area is $1 / 12$ for all periods. The observing periods are defined by the lunar cycle and do not always strictly match the given month, which is given for an easier identification.

rocking angle $\left(52^{\circ}\right)$ and distance from the Sun $\left(5^{\circ}\right)$ were applied. For the binned maximumlikelihood spectral analysis, the instrument response functions P7REP_SOURCE_V15 were used, together with the publicly available standard Isotropic and Galactic diffuse emission background models iso_source_v05.txt and gll_iem_v05 rev1.fit.

$1 \mathrm{ES} 0229+200$ is detected with a significance of $10.5 \sigma$ in the energy range $100 \mathrm{MeV}-500 \mathrm{GeV}$, compared to the $6.7 \sigma$ between 1 and $300 \mathrm{GeV}$ in [5]. The low flux in the Fermi-LAT band does not permit detailed temporal studies on monthly timescales. Nonetheless, the variability index ${ }^{4}$ for the monthly lightcurve has a value of 157 for 81 degrees of freedom, which indicates variability at the $5 \sigma$ level.

\subsection{X-rays}

In this work, X-ray data from Swift, RXTE and XMM-Newton are used ${ }^{5}$ (Fig. 1, third and fourth panels). The 30-days bin Swift-BAT lightcurve between 15 and $85 \mathrm{keV}$ is derived from the 15-days bin one in the 66 months Palermo BAT Catalog 6 . The 2008 and 2009 XMM-Newton and Swift-XRT, as well as the 2010 RXTE data are taken from [7], while the whole 2010-2011 RXTE lightcurve is taken from [13]. Finally, the most recent Swift-XRT data have been collected between the nights of October 1-2 and 10-11, 2013 during the aforementioned MWL campaign.

All the available Swift-XRT data collected between 2008 and 2015 (ObsIDs 0003124900100031249050 and 00080245001-00080245006) were analysed using the HEASoft software package v. $6.16^{7}$ with CALDB v. 20140120. All the events were cleaned and calibrated using the

${ }^{4} \mathrm{TS}_{\mathrm{var}}=2 \Sigma_{i}\left[\log \mathcal{L}_{i}\left(F_{i}\right)-\log \mathcal{L}_{i}\left(F_{\text {const }}\right)\right]$ where $F_{\text {const }}$ and $F_{i}$ are the average source flux and the flux of the $i$-th time bin. No limits on the bin significance are required.

${ }^{5} \mathrm{NUSTAR}$ data are not taken into account here since they are subject of a dedicated paper, in preparation.

${ }^{6}$ http://bat.ifc.inaf.it/bat_catalog_web/66m_bat_catalog.html

${ }^{7}$ http://heasarc.gsfc.nasa.gov/docs/software/lheasoft 
xrtpipeline task and the data in the $0.3-10 \mathrm{keV}$ energy range with grades $0-2$ for WT mode and 0-12 for PC mode were analysed. The lightcurve flux points were calculated from the spectra of single snapshots integrating between 2 and $10 \mathrm{keV}$. These were derived in the following way: the data were grouped using the grappha tool to have a minimum of 30 counts/bin and then fit using XSPEC v. 12.8.2 with a single power-law model and Galactic hydrogen absorption fixed to $n_{H}=8.06 \times 10^{20} \mathrm{~cm}^{-2}[14]$.

\subsection{Optical}

Optical monitoring of 1ES $0229+200$ has been carried out with the ATOM telescope [15] between 2007 and 2012. ATOM is a $75 \mathrm{~cm}$ altazimuth telescope equipped with an Apogee Alta U47 camera (Apogee Alta E47+ between 2004 and 2011) and works in robotic mode. It is part of the H.E.S.S. project and is used mainly for optical monitoring of variable $\gamma$-ray sources as a constant support for MWL observations. The observations for 1ES 0229+200 have been carried out typically once every three nights during the August-December visibility window. The standard reduction, the photometry and the source calibration are done with an automatic pipeline. The photometry uses an aperture of 4 ", while two reference stars in the vicinity of the source and present on the same frame are used for the calibration. During the 2013 MWL campaign ATOM was not operational and data were collected with the $70 \mathrm{~cm}$ telescope of the Abastunami Observatory (Georgia), equipped with an Apogee 6E camera. Observations taken with a R Cousins filter have been analysed with the Daophot II reduction software using an aperture diameter of $10^{\prime \prime}$.

\section{Results and discussion}

The Model analysis is more sophisticated and sensitive than the Hillas analysis [16] used in the previous publications. Its use permits therefore to have a much higher sensitivity to small flux variations. The monthly and yearly VHE lightcurves (top two panels of Fig. 1) show an emission which is not constant, neither on a yearly, nor on a monthly timescale. A fit to a constant value yields a $\chi^{2}$ of 84.3 (33.3) for 22 (7) degrees of freedom for the monthly (yearly) lightcurve. This translates in a probability of $5 \times 10^{-9}$ and $2 \times 10^{-5}$ respectively. The values for the fractional variability ([17], though whose errors are calculated as in [18]) are then $0.60 \pm 0.15$ and $0.39 \pm 0.11$. Variability is detected on a monthly timescale also between 0.58 to $1 \mathrm{TeV}$ and above $1 \mathrm{TeV}$ (probability of a constant flux of $1 \times 10^{-4}$ and $2 \times 10^{-5}$, respectively).

In 2009, H.E.S.S. and VERITAS observed the source contemporaneously (though on different nights). The time range spanned by the VERITAS observations was significantly longer than the H.E.S.S. one. Taking the windows into account, the measurements of both experiments are consistent with an increasing flux that reaches its maximum just before MJD 55150 and then decreases. The comparison of the VHE and X-ray data in 2009 [7] is very interesting. The source is in very low state for both H.E.S.S. and XMM-Newton during the MWL campaign (MJD 55065). This changes in the following three months, when Swift-XRT detects a factor $\sim 2$ flux increase [7] (also seen by Swift-BAT), which is mirrored by an enhancement in the emission in $\gamma$-rays of a factor 2 or 3 (Fig. 1). In all three wavebands, this state is followed by a decrease in the emission. Despite the limited sensitivity of Swift-BAT in the hard X-ray band, it is interesting to notice that the three periods of low VHE fluxes in 2008 and 2009 are mirrored also in this band. The R and B bands 


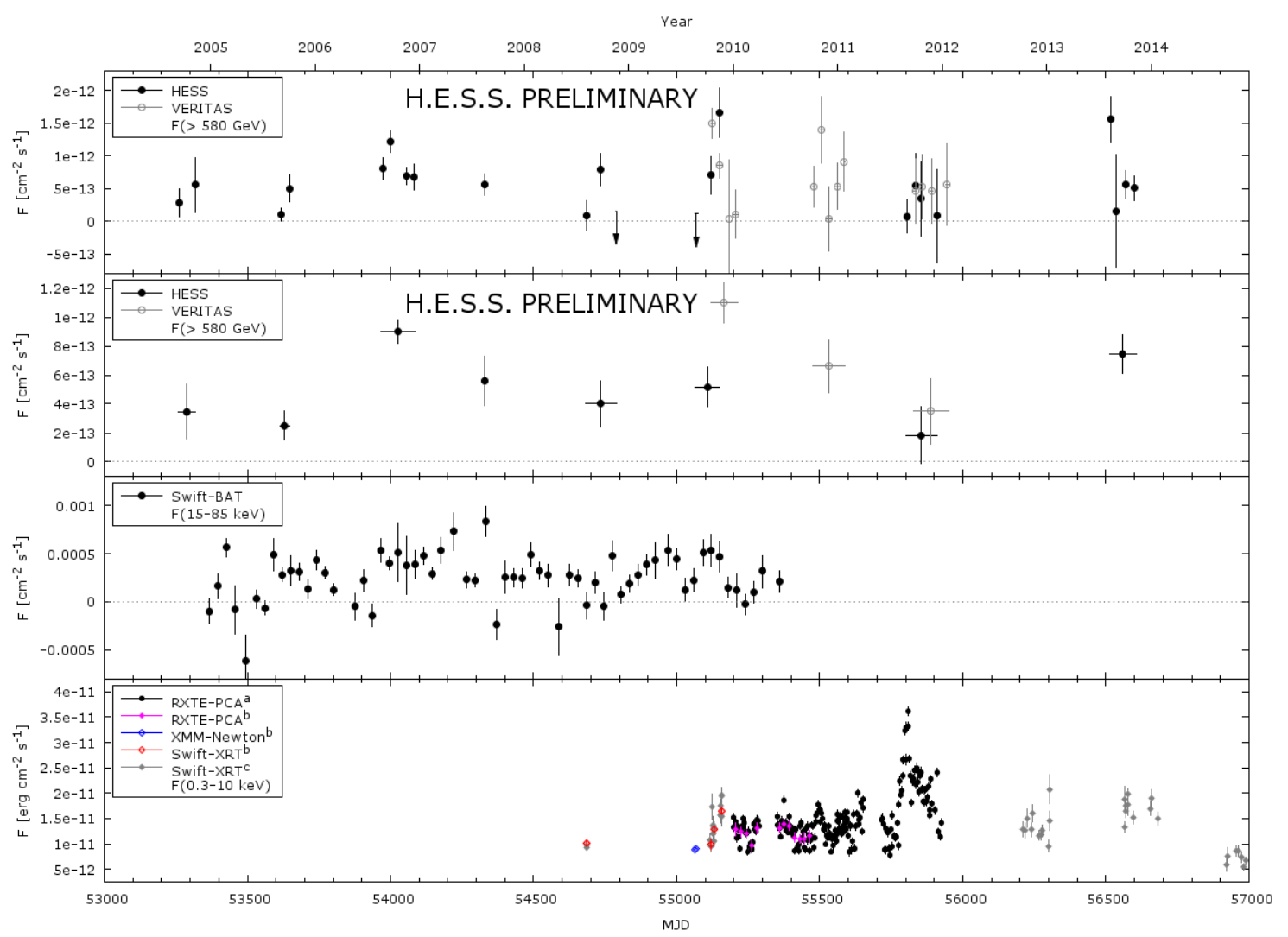

Figure 1: Lightcurves of the BL Lac object 1ES 0229+200 in different energy bands between 2004 and 2013. From top to bottom: monthly and yearly H.E.S.S. lightcurves above $580 \mathrm{GeV}$ - the VERITAS values from [8] are also depicted as comparison; hard X-rays monthly lightcurve between 15 and $85 \mathrm{keV}$ from Swift-BAT (the Palermo BAT Catalogue); soft X-ray lightcurve between 2 and $10 \mathrm{keV}$ for different instruments: ${ }^{a)}$ 2010-2012 RXTE dataset from [13], ${ }^{b}$ XMM-Newton, Swift-XRT and RXTE data from [7], ${ }^{c)}$ Swift-XRT data analysed in this work. The source is clearly variable in all energy bands. The discrepancy between the H.E.S.S. and VERITAS points in 2009 is explained by the non identical observation windows of the two instruments (see text). Three point at MJD 53831, 55331 and 55377 have been removed for clarity from the Swift-BAT plot because of their large negative fluxes or error bars.

optical lightcurves, instead, are constant within the errors and do not show any variations. During the second MWL campaign in 2013 (MJD 56570), 1ES 0229+200 seems to be relatively bright in $\mathrm{X}$-rays and on its average value in $\mathrm{TeV}$.

A correlation in the X-ray-VHE emission is actually expected by the SSC model, implying that the two components are generated by the same electron population. On the other hand, the big peak shown by RXTE in 2011 (MJD 55800) does not have a clear correspondence in TeV. This could be explained by the very low exposure in three out of the four periods of H.E.S.S. observations, or by a period of high activity of one of the other X-ray sources present in the field of view of RXTE (e.g. 1RXS J023558.0+201215, 1RXS J023427.5+192247 or one of the numerous XMM sources). 
A third possibility could be the presence of a second X-ray emitting zone, not related to any $\gamma$-ray emission. Finally, also Klein-Nishina suppression could play a role under certain conditions.

The confirmation of VHE variability on these timescales (already suggested in [8]) affects the determination of constrains on the IGMF. Focusing on the detected variability, if the $\sim 600 \mathrm{GeV}$ emission consists of secondary photons ("maximal" case in [4]), then a measurement of the IGMF can actually be done. Extrapolating Fig. 2 of [4] to shorter time delays, one measures the IGMF to be $\mathrm{B}_{\mathrm{IGMF}} \sim 3 \times 10^{-16} \mathrm{G}$.

Although it has been stated that $\mathrm{TeV} \gamma$-rays from 1ES $0229+200$ could be explained by proton cascade emission (e.g. [19]), the variability above $1 \mathrm{TeV}$ implies that the radiation of this source is dominated by primary $\gamma$-rays. Future studies investigating further the energy dependendent variability will allow the contribution of the cascade emission to the flux to be probed more deeply for both hadronic and leptonic models.

\section{Conclusion}

The 10 years long H.E.S.S. monitoring of the blazar 1ES 0229+200 between 2004 and 2013 has been presented for the first time in a MWL context. Clear variability is detected at VHE on monthly and yearly timescales. A hint of correlation between $\mathrm{TeV}$ and $\mathrm{X}$-ray emission comes from the contemporaneous observations with XMM-Newton and with Swift in 2009: the fluxes increase in all energy bands in similar way over four months. This supports an SSC emission model for the $\mathrm{X}$-ray and VHE emission in this source. The VHE monthly flux variability affects the derivation of lower limits on the IGMF. Assuming the emission at $\sim 600 \mathrm{GeV}$ to be of secondary photons, one can actually obtain a measurement of the IGMF of $\mathrm{B}_{\mathrm{IGMF}} \sim 3 \times 10^{-16} \mathrm{G}$.

\section{Acknowledgments}

The support of the Namibian authorities and of the University of Namibia in facilitating the construction and operation of H.E.S.S. is gratefully acknowledged, as is the support by the German Ministry for Education and Research (BMBF), the Max Planck Society, the German Research Foundation (DFG), the French Ministry for Research, the CNRS-IN2P3 and the Astroparticle Interdisciplinary Programme of the CNRS, the U.K. Science and Technology Facilities Council (STFC), the IPNP of the Charles University, the Czech Science Foundation, the Polish Ministry of Science and Higher Education, the South African Department of Science and Technology and National Research Foundation, and by the University of Namibia. We appreciate the excellent work of the technical support staff in Berlin, Durham, Hamburg, Heidelberg, Palaiseau, Paris, Saclay, and in Namibia in the construction and operation of the equipment.

This research has made use of the Palermo BAT Catalogue and database operated at INAF IASF Palermo, and of the lightcurves provided by the University of California, San Diego Center for Astrophysics and Space Sciences, X-ray Group (R.E. Rothschild, A.G. Markowitz, E.S. Rivers, and B.A. McKim), obtained at http://cass. ucsd.edu/ rxteagn/. 


\section{References}

[1] F. Aharonian et al., New constraints on the mid-IR EBL from the HESS discovery of VHE $\gamma$-rays from IES 0229+200, AEA 475 (2007) L9-L13, [arXiv : 0709 . 4584].

[2] F. Tavecchio et al., The intergalactic magnetic field constrained by Fermi/Large Area Telescope observations of the TeV blazar 1ES0229+200, MNRAS 406 (2010) L70-L74, [arXiv: 1004 . 1329].

[3] C. D. Dermer et al., Time Delay of Cascade Radiation for TeV Blazars and the Measurement of the Intergalactic Magnetic Field, ApJ 733 (2011) L21, [arXiv: 1011.6660].

[4] A. M. Taylor, I. Vovk, and A. Neronov, Extragalactic magnetic fields constraints from simultaneous GeV-TeV observations of blazars, AEA 529 (May, 2011) A144, [arXiv: 1101. 0932].

[5] I. Vovk et al., Fermi/LAT Observations of 1ES 0229+200: Implications for Extragalactic Magnetic Fields and Background Light, ApJ 747 (2012) L14, [arXiv: 1112 . 2534].

[6] M. Elvis et al., The Einstein Slew Survey, ApJS 80 (1992) 257-303.

[7] S. Kaufmann et al., IES 0229+200: an extreme blazar with a very high minimum Lorentz factor, AEA 534 (2011) A130, [arXiv: 1109.3628].

[8] E. Aliu et al., A Three-year Multi-wavelength Study of the Very-high-energy $\gamma$-Ray Blazar IES 0229+200, ApJ 782 (2014) 13, [arXiv: 1312.6592].

[9] F. Aharonian et al., Observations of selected AGN with HESS, AEA 441 (2005) 465-472, [astro-ph/0507207].

[10] M. de Naurois and L. Rolland, A high performance likelihood reconstruction of $\gamma$-rays for imaging atmospheric Cherenkov telescopes, Astroparticle Physics 32 (2009) 231-252, [arXiv: 0907 . 2610].

[11] D. Berge, S. Funk, and J. Hinton, Background modelling in very-high-energy $\gamma$-ray astronomy, AEA 466 (2007) 1219-1229, [astro-ph/0610959].

[12] F. Piron et al., Temporal and spectral gamma-ray properties of Mkn 421 above 250 GeV from CAT observations between 1996 and 2000, AEGA 374 (2001) 895-906, [astro-ph/0106196].

[13] E. Rivers, A. Markowitz, and R. Rothschild, Full Spectral Survey of Active Galactic Nuclei in the Rossi X-ray Timing Explorer Archive, ApJ 772 (2013) 114, [arXiv: 1306. 4376].

[14] P. M. W. Kalberla et al., The Leiden/Argentine/Bonn (LAB) Survey of Galactic HI. Final data release of the combined LDS and IAR surveys with improved stray-radiation corrections, AEA 440 (2005) 775-782, [astro-ph/0504140].

[15] M. Hauser et al., ATOM - an Automatic Telescope for Optical Monitoring, Astronomische Nachrichten 325 (2004) 659-659.

[16] A. M. Hillas, Cerenkov light images of EAS produced by primary gamma, International Cosmic Ray Conference 3 (1985) 445-448.

[17] S. Vaughan et al., On characterizing the variability properties of X-ray light curves from active galaxies, MNRAS 345 (2003) 1271-1284, [astro-ph/0307420].

[18] J. Poutanen, A. A. Zdziarski, and A. Ibragimov, Superorbital variability of X-ray and radio emission of Cyg X-1 - II. Dependence of the orbital modulation and spectral hardness on the superorbital phase, MNRAS 389 (2008) 1427-1438, [arXiv: 0802 . 1391].

[19] W. Essey, O. Kalashev, A. Kusenko, and J. F. Beacom, Role of Line-of-sight Cosmic-ray Interactions in Forming the Spectra of Distant Blazars in TeV Gamma Rays and High-energy Neutrinos, ApJ 731 (Apr., 2011) 51, [arXiv: 1011.6340]. 